\title{
Molecular transport in viscoelastic materials: mechanistic properties and chemical affinities
}

\author{
J. A. Ferreira * Paula de Oliveira ${ }^{\dagger} \quad$ P. M. da Silva ${ }^{\ddagger} \quad$ Laurent Simon $^{\S}$
}

\begin{abstract}
The effects of mechanical properties and chemical affinities of materials on the transport of solutes were studied. Simulations show that the kinetics of permeant fluids in matrices depends on the rheological and chemical properties of the polymer. Fick's law failed to describe transport through viscoelastic materials because of the force exerted on the incoming fluid causing a delay. Reversible binding to immobilizing sites also retarded permeation of molecules. An integro-differential equation was applied to model transport in the presence of solute membrane interactions. While the differential part of the equation was represented by an elliptic operator, the integral part, composed of two integrals, described the contributions of stress and reversible binding. Using Laplace transforms, the steady-state flux and effective time constant were calculated. The latter parameter represents a statistical interpretation of the waiting time to achieve equilibrium in the system. The lag time, that defines the first moment when a detectable concentration is measured in a receiver cell, was also studied using multiple integration. Subsequent analyses revealed the dependence of the steadystate flux, the effective time constant, the lag time on the Young modulus, the viscosity and the binding/unbinding rates. The results presented in this paper make it possible to tune the mechanical and chemical properties to achieve a desired transport profile.
\end{abstract}

Keywords: partial integro-differential equation, viscoelasticity, Laplace transform

\section{Introduction}

\subsection{Applications of polymeric materials}

Polymers continue to play a pivotal role in applications, such as controlled release systems, chemical protective clothing and impermeable liners. In controlled release systems, these ma-

\footnotetext{
${ }^{*}$ CMUC-Department of Mathematics, University of Coimbra. E-mail:ferreira@mat.uc.pt.

${ }^{\dagger}$ CMUC-Department of Mathematics, University of Coimbra. E-mail:poliveir@mat.uc.pt.

${ }^{\ddagger}$ Instituto Politécnico de Coimbra, ISEC, DFM, Rua Pedro Nunes, 3030-199 Coimbra, Portugal. CMUC. E-mail:pascals@isec.pt.

${ }^{\S}$ Otto H. York Department of Chemical, Biological and Pharmaceutical Engineering, New Jersey Institute of Technology. E-mail:laurent.simon@njit.edu
} 
terials are used as carriers ([1]) and coatings designed to mask the taste of a medication ([2]). Delivery via the oral route is difficult for proteins produced in bioreactors because of enzymes present in the gastrointestinal tract. In addition, the short life of these molecules makes it necessary to administer several injections to maintain a therapeutic plasma concentration. The ideal situation would be to shield the drug until it reaches the target site, at which point the carrier is able to unload its cargo in a predetermined manner. Poly(lactic-co-glycolic acid), or PLGA, and hydrogels are matrices used to protect the drug and help ensure a targeted delivery rate profile. Protective clothing is important to guard against chemical hazards or biohazards. Examples of these occurrences are nuclear disasters or war scenarios. Some of these compounds are absorbed through chemical protective clothing permeation, the skin barrier and the viable epidermis followed by distribution into the blood capillaries resulting in systemic poisoning. Nanotechnology based materials are investigated for providing protection to military personnel ([3]). Contrary to controlled release technologies, efforts are devoted to developing a strong barrier to prevent the absorption of chemicals. Impermeable liners, such as geomembranes, are designed to reduce risks associated with the diffusion of pollutants in the environment $([4])$. To serve as effective sealants against contaminants, the mechanical properties of the membrane have to be well understood. Studies should also shed light on how chemicals are being transported through these polymeric sheets ([4]) to prevent seepage of pollutants.

\subsection{The role of mathematical modeling in polymer research}

Advances in polymer science have resulted in the development of new products that administer therapeutic agents to the desired location in a timely manner. In spite of the notable success and progress, the lack of adequate mathematical models that account for the permeant/polymer structures limits further development in key research areas. A measure of the time it takes to achieve a steady-state condition (e.g. time constant) is sometimes missing from the literature or not written as an explicit function of the system characteristics. As a result, trial and error experiments are conducted to study the effects of critical parameters on dynamic behavior. These trials can be costly and may delay the time to market of a particular product.

Mathematical modeling and experimentation are essential to ensure sustainable development in the aforementioned polymer technologies. The approach allows researchers to link properties of a controlled release device, or a geomembrane, to its performance. Besides the mechanical aspects, chemical properties can be also used to control molecular diffusion. The inclusion of reversible binding sites in a carrier micro structure is currently used to slow the release of a solvent. In this framework, the influence of factors, such as the diffusion coefficient, viscoelasticity and binding/unbinding rates, can be readily assessed in a simulation environment before transitioning to a lab testing phase. The viscoelastic and the reactive 
behavior of the material and their influence on diffusive transport can be considered when theory guided experiments are carried out.

The transport of a fluid through a viscoelastic reactive matrix depends not only on the properties of the fluid but also on the mechanistic and chemical properties of the material. As a response to the strain caused by permeation, the matrix offers a resistance in the form of a stress exerted on the incoming fluid. This means that the brownian motion, underlying Fickian diffusion, is delayed by the viscoelasticity of the matrix. If the material is reactive, as it is the case when reversible binding to immobilizing sites takes place, an additional delay occurs in the solvent transport. The whole problem can be modeled by an integrodifferential equation, where the differential part represents the Fickian diffusion. The two integral operators in the equation are memory terms that describe the viscoelastic behavior and the chemical properties of the material.

Researchers presented a model that described diffusion through a viscoelastic material ([5]) where the mechanical behavior was represented by a Maxwell or a Maxwell/Voigt model and no chemical effects were considered. In [6] and [7], the chemical behavior of the matrix was investigated but no viscoelastic effects were included. Diffusion through a viscoelastic reactive material is analyzed in this work. A Maxwell generalized model ([8]) is used to examine the viscoelastic effects. The viscoelastic behavior is coupled with the binding of solvent to immobilizing sites in the matrix.

The theory of Laplace transforms is employed to characterize the steady-state flux and an effective time constant that can be interpreted statistically as the waiting time to achieve equilibrium in the system. The interesting point of the approach is that closed expressions for the flux and the equilibrium time can be deduced without solving the integro-differential equation. An expression for the lag time is established using multiple integration in space and time ([9], [10], [11], [12]). The dependence of the steady-state flux, the effective time constant and the lag time on the mechanical and chemical parameters of the model is fully analyzed. These findings are important tools for designing a system that behaves in a predefined manner.

Section 2 of this contribution focuses on an integro-differential model of transport through a viscoelastic membrane. The effective time constant is derived and compared to the lag time approach to estimate the period elapsed before reaching a steady-state flux. In Section 3 the joint influence of viscoelasticity and reversible binding is discussed. Numerical simulations in Sections 2 and 3 were performed with an implicit explicit finite difference method. Conclusions are drawn in Section 4.

\section{Diffusion and viscoelastic properties}

\subsection{Mathematical model}

The diffusion of a penetrant in a viscoelastic membrane $\Omega \subset \mathbb{R}^{3}$ is described by 


$$
\left\{\begin{array}{l}
\frac{\partial u}{\partial t}=\nabla \cdot(D \nabla u)+\nabla \cdot\left(D_{v} \nabla \sigma\right), \text { in } \Omega, t>0 \\
\sigma=-\int_{0}^{t}\left(E_{0+} \sum_{1}^{n} E_{i} e^{-\frac{t-s}{\tau_{i}}}\right) \frac{\partial \epsilon}{\partial s} d s, \text { in } \Omega, t>0
\end{array}\right.
$$

where $u$ denotes the concentration of the penetrant; $D$ and $D_{v}$ correspond to the diffusion and stress-driven diffusion tensors, respectively. The relationship between the stress $\sigma$ and the strain $\epsilon$ in a viscoelastic material is described by a Maxwell generalyzed model with $\mathrm{n}$ arms. Each contains one elastic spring (Figure 1). In Eq. (1), $E_{0}$ is the Young modulus of the

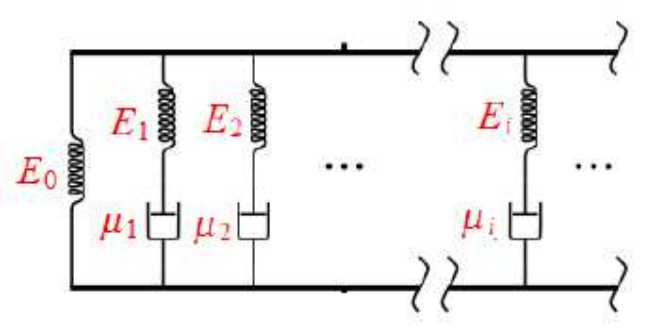

Figure 1: Maxwell generalyzed model representation.

isolated spring; $E_{i}, i=1, \ldots, n$, is the Young Modulus of the Maxwell arms; $\tau_{i}$ represents the relaxation time of Maxwell arm $i$ and is defined by $\tau_{i}=\frac{\mu_{i}}{E_{i}} ; \mu_{i}$ is the viscosity of the material. The units used in Eq. (1) are presented in Annex I. The minus sign, in the formulation of the stress, indicates that $\sigma$ and $\epsilon$ are of opposite sign. This is due to the fact that the polymer forms a barrier to the entry of the penetrant fluid by exerting on it a stress $\sigma$ in response to the deformation $\epsilon$ the fluid causes in the matrix. When $\Omega=[0, L]$, the viscoelastic diffusion coefficient $D_{v}$, has a precise physical interpretation and it can be proved that $D_{v}>0$ ([13], [14]). In $[5,15,16]$, the authors considered $D_{v}<0$ and the stress $\sigma$ with the same sign as the deformation $\epsilon$. Their approach is similar to the model discussed in this work.

System (1) is completely defined by the following initial conditions:

$$
u(x, 0)=0, \sigma(x, 0)=0, x \in \Omega
$$

and boundary conditions:

$$
\left\{\begin{array}{l}
u(x, t)=u_{0}, \sigma(x, t)=-\widetilde{E}_{0} u_{0}, x \in \Gamma_{0} \\
u(x, t)=0, \sigma(x, t)=0, x \in \Gamma_{L},
\end{array}\right.
$$

with $\widetilde{E}_{0}=k E_{0}$, where $k$ is a dimensionalized constant defined by $\epsilon=k u([15],[16],[17],[18])$. $\Gamma_{0}$ stands for the inlet boundary in contact with the penetrant source and $\Gamma_{L}$ the membranereceiver interface. 
Replacing the stress $\sigma$ into the first equation of (1) and assuming that $D$ and $D_{v}$ are constants we have

$$
\frac{\partial u}{\partial t}=\left(D-\widetilde{D_{v}} E_{0}-\widetilde{D_{v}} \sum_{i=1}^{n} E_{i}\right) \Delta u+\widetilde{D_{v}} \int_{0}^{t} \sum_{i=1}^{n} \frac{E_{i}}{\tau_{i}} e^{-\frac{t-s}{\tau_{i}}} \Delta u(x, s) d s .
$$

$\Omega$ is an homogeneous domain and $\widetilde{D_{v}}=k D_{v}$. In what follows, we drop the tilde in $D_{v}$. Equation (4) can then assume the conservative form

$$
\frac{\partial u}{\partial t}=-\nabla . J
$$

with the flux $J$ defined by

$$
J(x, t)=-\left(D-D_{v} E_{0}-D_{v} \sum_{i=1}^{n} E_{i}\right) \nabla u-D_{v} \int_{0}^{t} \sum_{i=1}^{n} \frac{E_{i}}{\tau_{i}} e^{-\frac{t-s}{\tau_{i}}} \nabla u(x, s) d s .
$$

Note that $D_{v}=0$ leads to a pure Fickian diffusion. When $D_{v} \neq 0$, several well-known relationships based on the Maxwell generalized model can be developed ([8]). The standard linear solid model is obtained when the system is described by an isolated spring and a single Maxwell arm. Without the arm, the material behaves like a pure elastic solid, leading to

$$
\frac{\partial u}{\partial t}=\left(D-D_{v} E_{0}\right) \Delta u,
$$

i.e., a pure Fickian diffusion problem with the diffusion coefficient defined by $D-D_{v} E_{0}$. The condition $D-D_{v} E_{0}>0$ must then be satisfied to have a well-posed system. This constraint is physically sound as it establishes that Fickian transport dominates the viscoelastic effects. With no springs and only one damper, a viscous fluid model is defined:

$$
\sigma=-\mu \frac{\partial \epsilon}{\partial t}
$$

In the case of two arms, one with a free spring and the other with a free damper, the Maxwell generalized model corresponds to the Kelvin Solid model with the stress $\sigma$ defined by

$$
\sigma=-\left(E_{0} \epsilon+\mu \frac{\partial \epsilon}{\partial t}\right)
$$

A Maxwell fluid model is obtained when $E_{0}=0$.

The viscoelastic behavior of System (1) with $n=1$ is plotted in Figure 2, for the domain $\Omega=\left[0,10^{-3}\right], u_{0}=1, L=10^{-3}, D=3 \times 10^{-3}, D_{v}=10^{-6}, E_{0}=10^{3}, E_{1}=10$, and $\mu=10^{-5}$.

The behaviors of $u$ and the absolute value of $\sigma$ are qualitatively analogous as predicted by the second equation in (1) and the assumption $\epsilon=k u$. The concentration of the diffusing species increases inside the polymeric matrix and the highest stress is observed where and when the highest concentration is reached. 

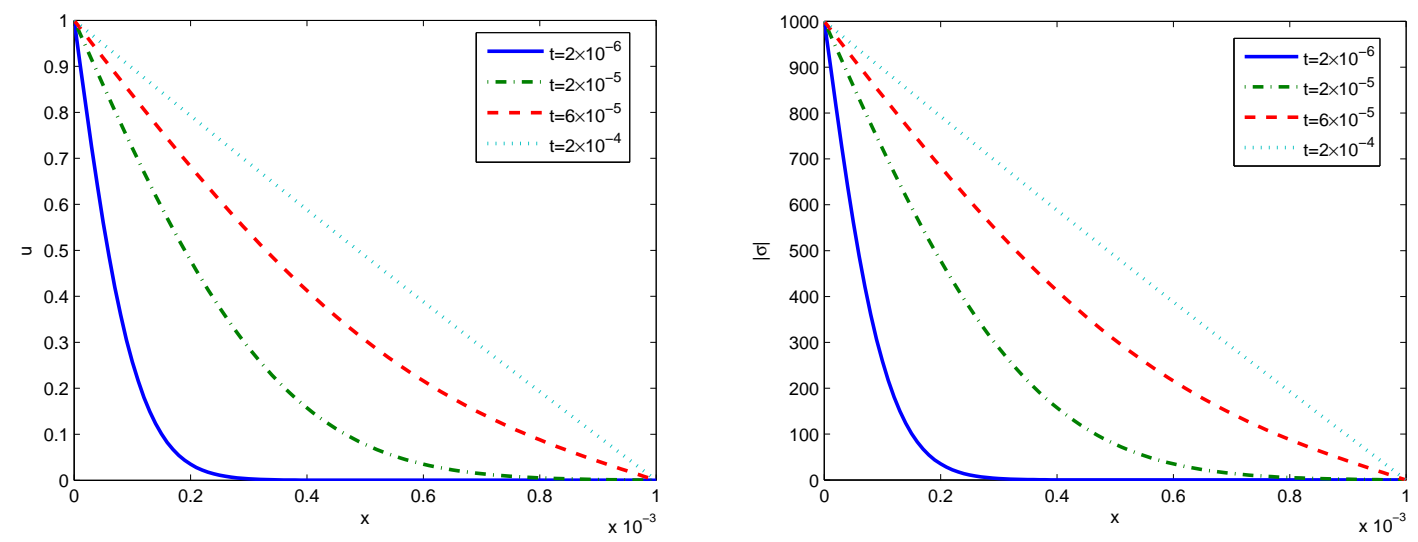

Figure 2: Behaviors of $u$ (left) and absolute value of $\sigma$ (right) for the Maxwell generalized model with $n=1$.

Figure 3 represents the flux at $x=L$ as defined by Eq. (6). For $D_{v} \neq 0$, there is a delay in the appearance of a detectable concentration at $x=L$. As $D_{v}$ increases, the steady-state flux decreases. The simulations were performed using a numerical implicit-explicit method implemented in Matlab® (The MathWorks, Inc.). The diffusion operator was discretized implicitly while the discretization of the integral operator was explicit.

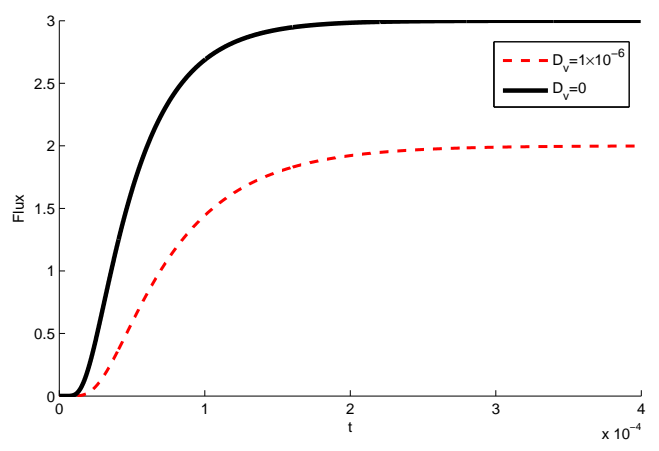

Figure 3: Behavior of the flux at $x=L$ for $D_{v}=0$ (pure diffusion) and $D_{v}=10^{-6}$ (Maxwell generalized model with $n=1$ ).

\subsection{Identification of Dynamic Characteristics}

Closed-form expressions for lag time $\left(t_{l a g}\right)$ and the effective time constant $\left(t_{\text {eff }}\right)$ are derived. The performance of these metrics has been investigated by several researchers ([5], [7], [9], $[10],[19],[20],[21])$. Simulations are conducted to study how accurately they predict the 
steady-state flux.

\subsubsection{Lag time}

The lag time monitors the first appearance of the penetrant: the moment when a detectable concentration is measured in a receiver cell at $x=L$. This method is often applied to determine the model parameters using the cumulative amount of penetrant released versus the time plot. The lag time for problem (1) is computed in the one-dimensional case.

Let $D^{*}=D-D_{v} E_{0}-D_{v} \sum_{i=1}^{n} E_{i}$. Following the Frisch method ([7], [11], [12]), Eq. (5) is integrated twice in space and once in time, such that

$$
\int_{0}^{L} \int_{x}^{L} u(y, t) d y d x=-L Q(t)+\int_{0}^{t} \int_{0}^{L} J(x, s) d x d s
$$

where $Q(t)=\int_{0}^{t} J(L, s) d s$. From Eqs. (6) and (7) we have

$$
\begin{aligned}
\int_{0}^{L} \int_{x}^{L} u(y, t) d y d x= & -L Q(t)-\int_{0}^{t} \int_{0}^{L} D^{*} \frac{\partial u}{\partial x}(x, s) d x d s \\
& -D_{v} \int_{0}^{t} \int_{0}^{L} \int_{0}^{s} \sum_{i=1}^{n} \frac{E_{i}}{\tau_{i}} e^{-\frac{s-\tau}{\tau_{i}}} \frac{\partial u}{\partial x}(x, \tau) d \tau d x d s .
\end{aligned}
$$

As

$$
-\int_{0}^{t} \int_{0}^{L} D^{*} \frac{\partial u}{\partial x}(x, s) d x d s=D^{*} u_{0} t
$$

and

$$
\int_{0}^{t} \int_{0}^{L} \int_{0}^{s} \sum_{i=1}^{n} \frac{E_{i}}{\tau_{i}} e^{-\frac{s-\tau}{\tau_{i}}} \frac{\partial u}{\partial x}(x, \tau) d \tau d x d s=-u_{0}\left(t \sum_{i=1}^{n} E_{i}+\sum_{i=1}^{n} E_{i} \tau_{i} e^{-\frac{t}{\tau_{i}}}-\sum_{i=1}^{n} E_{i} \tau_{i}\right)
$$

we conclude from Eqs. (8)-(10) that

$$
Q(t)=-\frac{1}{L}\left(\int_{0}^{L} \int_{x}^{L} u(y, t) d y d x-D^{*} u_{0} t-D_{v} u_{0}\left(t \sum_{i=1}^{n} E_{i}+\sum_{i=1}^{n} E_{i} \tau_{i} e^{-\frac{t}{\tau_{i}}}-\sum_{i=1}^{n} E_{i} \tau_{i}\right)\right) .
$$

To monitor the first appearance of the penetrant at $x=L$ the first instant $t^{*}$ should be computed such that

$$
Q\left(t^{*}\right) \neq 0
$$


We estimate $t^{*}$ by $t_{\text {lag }}$ which is the solution of $Q_{a}(t)=0$, where $y=Q_{a}(t)$ is the equation of the asymptote of $y=Q(t)$. The following equation is obtained from Eq. (11)

$$
\int_{0}^{L} \int_{x}^{L} u^{S}(y) d y d x=t_{l a g} u_{0}\left(D-D_{v} E_{0}\right)-D_{v} u_{0} \sum_{i=1}^{n} E_{i} \tau_{i},
$$

where $u^{S}$ represents the steady state of $u$.

To determine $u^{S}$ the Laplace transform is applied to Eq. (1):

$$
\bar{u}(x, p)=\frac{u_{0}}{p} \frac{\sinh \delta(L-x)}{\sinh \delta L},
$$

where $\bar{u}(x, p)$ represents the Laplace transform of $u(x, t), \bar{u}(x, p)=\int_{0}^{\infty} e^{-p t} u(x, t) d t$,

$$
\delta= \pm \sqrt{\frac{p}{D+D_{v} \bar{k}(p)-D_{v} E_{0}+D_{v} \sum_{i=1}^{n} E_{i}}}
$$

and

$$
\bar{k}(p)=\sum_{i=1}^{n} E_{i} \frac{1}{\tau_{i} p+1} .
$$

Application of the Final Value Theorem leads to $u^{s}(x)=\lim _{p \rightarrow 0} \bar{u}(x, p)$ and consequently

$$
u^{S}(x)=u_{0} \frac{L-x}{L} .
$$

Replacing the expression of $u^{s}(x)$ in Eq. (12) results in

$$
t_{l a g}=\frac{\frac{L^{2}}{6}+D_{v} \sum_{i=1}^{n} E_{i} \tau_{i}}{\left(D-D_{v} E_{0}\right)} .
$$

The constraint $D-D_{v} E_{0}>0$, is imposed in order to have a positive $t_{\text {lag }}$. Therefore, the the Fickian contribution dominates the process and there is an effective penetration of the fluid in the polymer. After substituting $\tau_{i}=\frac{\mu_{i}}{E_{i}}$ in Eq. (16), it is clear that the lag time is independent of the Young modules of the Maxwell arms. It is an increasing function of Young modulus $E_{0}$ of the free spring and the global viscosity $\sum_{i=1}^{n} \mu_{i}$. In this sense, the Maxwell generalized model is equivalent to a Kelvin solid model.

Figure 4 shows $t_{l a g}$ as a function of $E_{0}$. A Maxwell generalized model with $n=1$ was used. As $E_{0}$ increases, the steady-state flux decreases and the lag time increases. The role of $E_{0}$ is to lower the effective diffusion coefficient of the molecules in the membrane. The crosslink density of polymer can be quantified by $\frac{E_{0}}{3 R T}$, where $R$ is the universal constant and $T$ is the absolute temperature. As $E_{0}$ increases, the crosslink density increases, which leads to a large degree of entanglement in the polymer. The net effect of this change is a delay in the transport of the solute. 


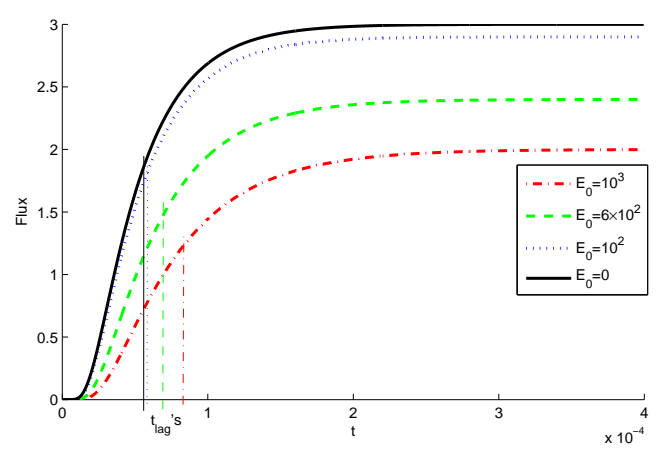

Figure 4: Behavior of the flux and $t_{l a g}$ for different $E_{0}$.

\subsubsection{Effective time constant}

The effective time constant indicates the time elapsed before reaching a steady-state condition. This measure has been first proposed in [20] and lately addressed in [5], [9], [19] and [22].

Following [20], $t_{\text {eff }}$, is calculated by

$$
t_{e f f}=\frac{\int_{0}^{\infty}\left(J(L, s)-J^{S}\right) s d s}{\int_{0}^{\infty} J(L, s)-J^{S} d s}
$$

where $J^{S}$ represents the steady flux.

While the lag time monitors the first appearance of the diffusing solvent, $t_{\text {eff }}$ measures the onset of an equilibrium state. In fact, it can be proved, using a statistical interpretation ([5]), that for $t=4 t_{\text {eff }}$, we have $J\left(L, 4 t_{\text {eff }}\right)=98.17 \% J^{S}(x)$ for a first-order system.

Equation (17) can be applied without explicit knowledge of the analytical form of the flux. As pointed out in [20], if the Laplace transform of $J(L, t), \bar{J}(L, p)$, can be written as

$$
\bar{J}(L, p)=\frac{1}{p}\left(B_{1}+B_{2} p+B_{3} p^{2}\right)+\ldots
$$

for $p$ small and where $B_{2} \neq 0$, then

$$
t_{\text {eff }}=-\frac{B_{3}}{B_{2}}
$$

The steady-state flux can be obtained from the Final Value Theorem. As

$$
\bar{J}(x, p)=-\left(D-D_{v} E_{0}-D_{v} \sum_{i=1}^{n} E_{i}\right) \frac{\overline{\partial u}}{\partial x}-D_{v} \sum_{i=1}^{n} \frac{E_{i}}{\tau_{i}} \frac{1}{p+\frac{1}{\tau_{i}}} \frac{\overline{\partial u}}{\partial x},
$$

we have from Eqs. (13) and (20)

$$
\bar{J}(L, p)=\left(D-D_{v} E_{0}-D_{v} \sum_{i=1}^{n} E_{i}+D_{v} \sum_{i=1}^{n} \frac{E_{i}}{\tau_{i}} \frac{1}{p+\frac{1}{\tau_{i}}}\right) u_{0} \frac{\delta}{\sinh \delta L},
$$


where $\delta$ is defined in Eq. (14). Eq. (21) can be written in the form:

$$
\bar{J}(L, p)=-\frac{u_{0}}{p L} \frac{1}{\frac{1}{w}+\frac{p}{w^{2}} \frac{L^{2}}{3 !}+\frac{p 2}{w^{3}} \frac{L^{4}}{5 !} \cdots},
$$

with

$$
w(p)=D-D_{v} E_{0}-D_{v} \sum_{i=1}^{n} E_{i}+D_{v} \sum_{i=1}^{n} \frac{E_{i}}{\tau_{i}} \frac{1}{p+\frac{1}{\tau_{i}}} .
$$

Using the Final Value Theorem the steady-state flux becomes

$$
J^{S}(L)=\frac{u_{0}}{L}\left(D-D_{v} E_{0}\right)
$$

Therefore, $J^{S}(L)$ is a decreasing function of $E_{0}$ and, consequently, of the crossslink density, which is a physically sound result. It can also be deduced from Eq. (24) that the condition

$$
D>D_{v} E_{0}
$$

should hold in order to have a release of the penetrant.

The effective time constant is calculated from Eqs. (18)-(23):

$$
t_{e f f}=\frac{1}{D-D_{v} E_{0}} \frac{D_{v}\left(D-D_{v} E_{0}\right) \sum_{i=1}^{n} \mu_{i} \tau_{i}+\frac{7 L^{4}}{360}}{D_{v} \sum_{i=1}^{n} \mu_{i}+\frac{L^{2}}{3 !}} .
$$

When the relaxation times $\tau_{i}$ are replaced by $\mu_{i} / E_{i}$, it is clear that $t_{\text {eff }}$ is a function of the Young modulus $E_{i}$ of the Maxwell arms. By comparison, note that the lag time is insensitive to $E_{i}$. We recall that at $t=0$ the Young modulus of the material is represented by $E_{0}+\sum_{i=1}^{n} E_{i}$, while at steady state it is defined by $E_{0}$. The effective time constant increases with $\tau_{i}$.

The behavior of the flux at $L$ is shown in Figure 5 for the same parameters used in Figure 2. The steady-state flux, calculated from Eq. (24), is 2 as depicted in the figure. Also, $t_{\text {lag }}=8.3338 \times 10^{-5}$ and $4 t_{\text {eff }}=2.3332 \times 10^{-4}$ are shown in the plot. In this case $J\left(4 t_{\text {eff }}\right)=$ 1.9593, which corresponds to $98 \% \times 2=1.96$. If we interpret ([23]) Eq. (17) as the first moment associated with a density probability function and making the natural ansatz that the density is exponentially shaped, the following prediction is obtained $J\left(4 t_{e f f}\right)=\left(1-e^{-4}\right) J^{S}$. This shows that our estimate is accurate. As $J\left(t_{\text {lag }}\right) \geq 50 \% J^{S}$, we conclude that $t_{\text {lag }}$ provides a poor estimate for the first appearance of penetrant at $x=L$.

The influence of $E_{0}$ is illustrated in Figure 6. As $E_{0}$ increases, $J^{S}$ decreases (Eq.(24)) and $t_{e f f}$ increases (Eq.(25)). The steady-state flux is not very sensitive to variations in $E_{1}$ (Figure 7 , left panel) and in $\mu$ (Figure 7 , right panel). As a result, $E_{0}$ is the only significant mechanistic parameter. 


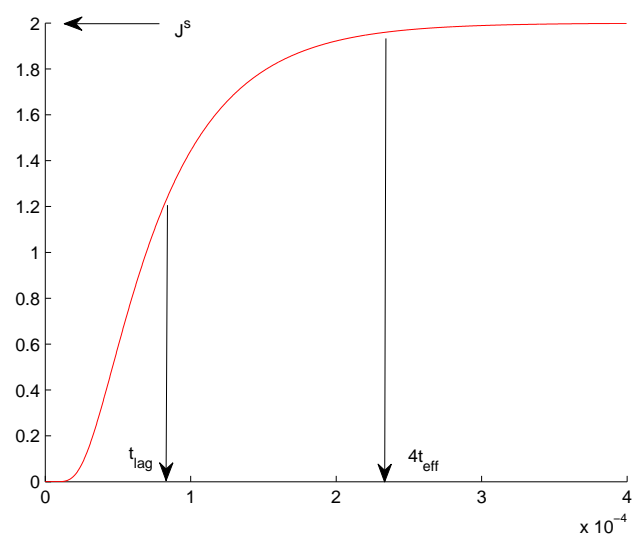

Figure 5: Behavior of flux for Maxwell generalized with $n=1$.

\section{A General Mechanistic-Chemical Model}

\subsection{Mass Flux}

The transport of a solute across a viscoelastic membrane and in the presence of a chemical reaction is described in this section. While the stress function is still described by the generalized Maxwell model, the penetrant is allowed to bind reversibly to the viscoelastic matrix:

$$
\left\{\begin{array}{l}
\frac{\partial u}{\partial t}=\nabla \cdot(D \nabla u)+\nabla \cdot\left(D_{v} \nabla \sigma\right)+f(u, v), \text { in } \Omega, t>0 \\
\frac{\partial v}{\partial t}=g(u, v), \text { in } \Omega, t>0 \\
\sigma=-\int_{0}^{t}\left(E_{0+} \sum_{i=1}^{n} E_{i} e^{-\frac{t-s}{\tau_{i}}}\right) \frac{\partial \epsilon}{\partial s} d s, \text { in } \Omega, t>0
\end{array}\right.
$$

where $u$ represents the free penetrant concentration, $v$ the bound penetrant concentration, $D$ the diffusion coefficient and $D_{v}$ the viscoelastic diffusion coefficient. The function $f$ stands for the affinity between the penetrant and the polymer and $g=-f$.

The system is coupled with the following initial conditions:

$$
u(x, 0)=0, v(x, 0)=0, \sigma(x, 0)=0, x \in \Omega
$$

and boundary conditions

$$
\left\{\begin{array}{l}
u(0, t)=u_{0}, \sigma(x, t)=-k E_{0} u_{0}, v(x, t)=\frac{\lambda_{2}}{\lambda_{1}} u_{0}\left(1-e^{-\lambda_{1} t}\right), x \in \Gamma_{0} \\
u(x, t)=0, \sigma(x, t)=0, x \in \Gamma_{L}
\end{array},\right.
$$

where, as before, $\Gamma_{0}$ represents the inlet boundary and $\Gamma_{L}$ the outlet boundary. 


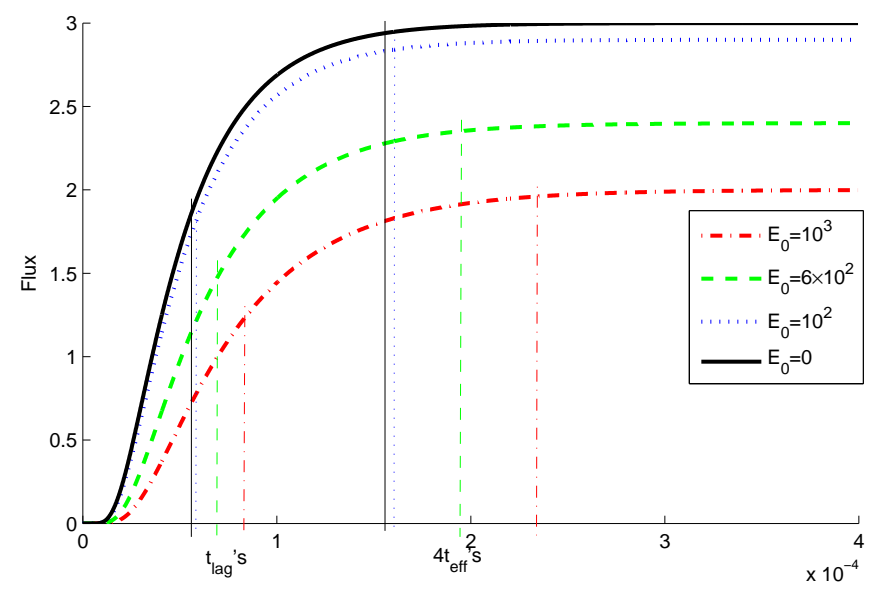

Figure 6: Influence of $E_{0}$ for Maxwell generalized with $n=1$.
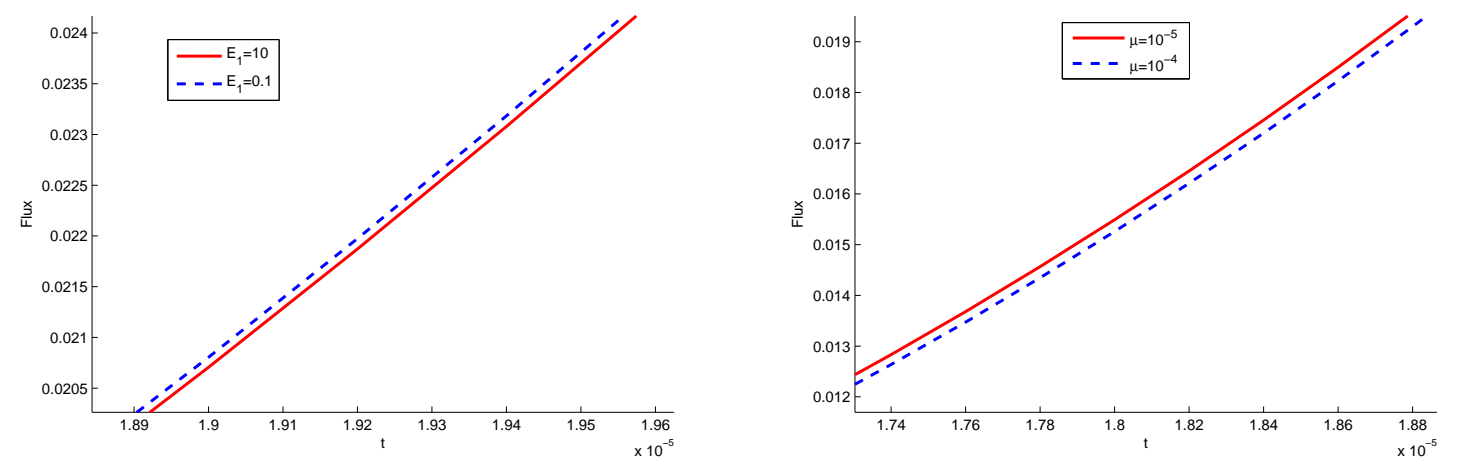

Figure 7: Influence of $E_{1}$ and $\mu$ for Maxwell generalized with $n=1$.

The function $f$ can be written as a linear function of form:

$$
f(u, v)=-\lambda_{2} u+\lambda_{1} v
$$

where $\lambda_{1}$ and $\lambda_{2}$ are the unbinding and binding rates, respectively ([7]).

Assuming that $\epsilon=u, D$ and $D_{v}$ constants, the following equation is obtained after using Eq. (29):

$$
\frac{\partial u}{\partial t}=D \Delta u-D_{v} \int_{0}^{t}\left(\sum_{i=1}^{n} E_{i} e^{-\frac{t-\tau}{\tau_{i}}}+E_{0}\right) \frac{\partial \Delta u}{\partial \tau}(x, \tau) d \tau+\lambda_{1} \lambda_{2} \int_{0}^{t} e^{-\lambda_{1}(t-\tau)} u(x, \tau) d \tau-\lambda_{2} u
$$

The flux becomes 


$$
J(x, t)=-D \nabla u+D_{v} \int_{0}^{t}\left(\sum_{i=1}^{n} E_{i} e^{-\frac{t-s}{\tau_{i}}}+E_{0}\right) \frac{\partial \nabla u}{\partial s}(x, s) d s .
$$

Using the Final Value Theorem, $J^{S}$ can be written as

$$
J^{s}=\frac{u_{0}}{L}\left(D-D_{v} E_{0}\right)
$$

which means that the steady-state flux is not sensitive to the reaction rates, as expected.

\subsection{Lag time}

The lag time is computed by first writing the temporal changes in $u$ and $v$ as

$$
\left\{\begin{array}{l}
\frac{\partial u}{\partial t}=-\frac{\partial J}{\partial x}+f(u, v), x \in \Omega, t>0 \\
\frac{\partial v}{\partial t}=-f(u, v), x \in \Omega, t>0
\end{array}\right.
$$

where $J$ is defined in (31). From (33) we have

$$
\frac{\partial u}{\partial t}+\frac{\partial v}{\partial t}=-\frac{\partial J}{\partial x}
$$

Following the Frisch method, the following equation is obtained:

$$
\int_{0}^{L} \int_{x}^{L}\left(\frac{\partial u}{\partial t}(y, t)+\frac{\partial v}{\partial t}(y, t)\right) d y d x=-L J(L, t)+\int_{0}^{L} J(x, t) d x .
$$

Changing the order of integration in the expression on the left hand side of the last equation we have

$$
\int_{0}^{L} x\left[\frac{\partial u}{\partial t}(x, t)+\frac{\partial v}{\partial t}(x, t)\right] d x=-L J(L, t)+\int_{0}^{L} J(x, t) d x
$$

and integrating in time gives

$$
\int_{0}^{L} x[u(x, t)+v(x, t)] d x=-L Q(t)+\int_{0}^{t} \int_{0}^{L} J(x, \tau) d x d \tau .
$$

To compute the asymptote of $Q(t)$, we calculate the steady state of $\mathrm{u}$ and $\mathrm{v}$ using the Final Value Theorem. The expression of $u^{s}$ is the same as in Eq. (15). From Eqs. (31) and (26) we have

$$
v(x, t)=\lambda_{2} \int_{0}^{t} e^{-\lambda_{1}(t-\tau)} u(x, \tau) d \tau .
$$

The Laplace transform of $v$ is

$$
\bar{v}(x, p)=\lambda_{2} \frac{1}{p+\lambda_{1}} \bar{u}(x, p)
$$


that is

$$
\bar{v}(x, p)=u_{0} \lambda_{2} \frac{1}{p\left(p+\lambda_{1}\right)} \frac{\sinh \widehat{\delta}(L-x)}{\sinh \widehat{\delta} L},
$$

where $\widehat{\delta}$ is defined by

$$
\widehat{\delta}^{2}=\frac{p\left(p+\lambda_{1}+\lambda_{2}\right)}{\left(p+\lambda_{1}\right) w}
$$

and $w$ is defined in Eq. (23). The Final Value Theorem applied to Eq. (35) results in

$$
v^{S}(x)=u_{0} \frac{\lambda_{2}}{\lambda_{1}} \frac{L-x}{L} .
$$

The lag time is calculated from Eqs (34), (35) and the expression of $v^{s}(x)$ :

$$
t_{\text {lag }}=\frac{\left(1+\frac{\lambda_{2}}{\lambda_{1}}\right) \frac{L^{2}}{6}+D v \sum_{i=1}^{n} E_{i} \tau_{i}}{\left(D-D_{v} E_{0}\right)} .
$$

When $D_{v}=0$ we obtain $t_{\text {lag }}=\left(1+\frac{\lambda_{2}}{\lambda_{1}}\right) \frac{L^{2}}{6 D}$ as referred in [7].

The lag time is insensitive to the reaction rates for a constant $\frac{\lambda_{2}}{\lambda_{1}}$, as observed in [7], for reactive membranes under no mechanical stress. However this is not an accurate prediction because $Q(t)$ depends on the binding rate $\lambda_{2}([7])$.

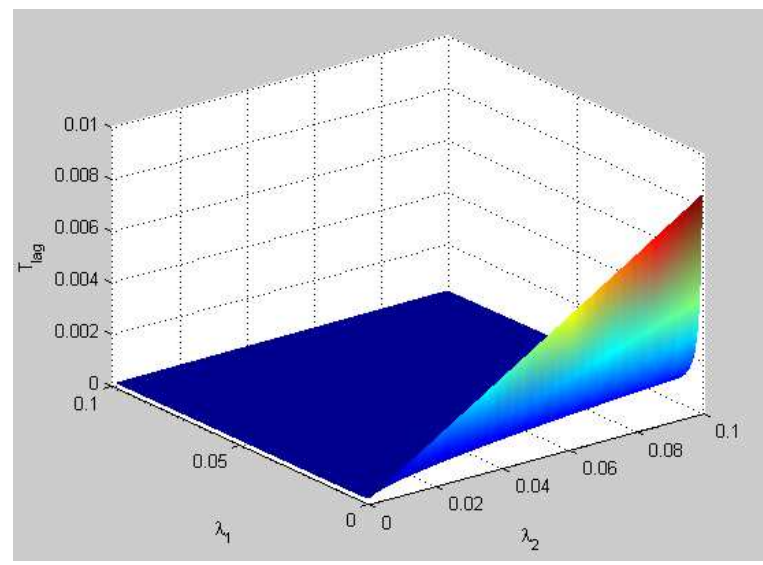

Figure 8: Influence of chemical reaction in $t_{\text {lag }}\left(\Omega=\left[0,10^{-3}\right], u_{0}=1, L=10^{-3}, D=\right.$ $\left.3 \times 10^{-3}, D_{v}=10^{-6}, E_{0}=10^{3}, E_{1}=10\right)$.

Figure 8 shows $t_{l a g}$ as a function of $\lambda_{1}$ and $\lambda_{2}$. As expected the lag time is an increasing function of $\lambda_{2}$ and a decreasing function of $\lambda_{1}$. These behaviors are clearly observed in Figure 8 for low values of the unbinding rate $\lambda_{1}$ and large values of the binding rate $\lambda_{2}$, respectively. 


\begin{tabular}{|c|c|c|c|}
\hline$\lambda_{1}$ & $t_{\text {eff }}$ & $4 t_{\text {eff }}$ & $\frac{J\left(4 t_{e f f}\right)}{J^{s}}$ \\
\hline \hline 10 & 0.0246 & 0.0984 & $98.236 \%$ \\
5 & 0.0277 & 0.1108 & $98.908 \%$ \\
1 & 0.1166 & 0.4664 & $99.784 \%$ \\
0.5 & 0.3613 & 1.4452 & $99.836 \%$ \\
0.1 & 5 & 20 & $99.956 \%$ \\
\hline
\end{tabular}

Table 1: Influence of $\lambda_{1}$ on the effective time and on the steady-state flux $\left(D=5 \times 10^{-4}, D_{v}=\right.$ $\left.0, \lambda_{1}=0.1, L=1 \times 10^{-2}, E_{1}=10\right)$.

\subsection{Effective time constant}

The effective time constant is derived using a procedure similar to the one implemented in Section 2.2.

From Eq. (31), the Laplace transform of $J$ is

$$
\bar{J}(L, p)=\frac{u_{0}}{p} w(p) \frac{\widehat{\delta}}{\sinh (\widehat{\delta} L)}
$$

where

$$
w(p)=D-D_{v} E_{0}-D_{v} \sum_{i=1}^{n} E_{i}+D_{v} \sum_{i=1}^{n} \frac{E_{i}}{p \tau_{i}+1}
$$

and

$$
\frac{w(p)}{p}=\frac{1}{\widehat{\delta}^{2}} \frac{p+\lambda_{1}+\lambda_{2}}{p+\lambda_{1}} .
$$

Expanding $\sinh (\widehat{\delta} L)$ as a series, in the neighborhood of $p=0, \bar{J}(L, p)$ takes the form given by Eq. (18). After some computations, $t_{\text {eff }}$ can be written as:

$$
t_{e f f}=\frac{1}{D-D_{v} E_{0}} \frac{\frac{7}{360}\left(\frac{\lambda_{1}+\lambda_{2}}{\lambda_{1}}\right)^{2} L^{4}+D_{v}\left(D-D_{v} E_{0}\right) \sum_{i=1}^{n} \eta_{i} \tau_{i}+\left(\frac{\lambda_{2}}{\lambda_{1}^{2}}\right) \frac{L^{2}}{3 !}\left(D-D_{v} E_{0}\right)}{D_{v} \sum_{i=1}^{n} \eta_{i}+\frac{L^{2}}{3 !} \frac{\lambda_{1}+\lambda_{2}}{\lambda_{1}}} .
$$

In this case, $t_{\text {eff }}$ depends on $\lambda_{1}$ and $\lambda_{2}$, and not only on $\frac{\lambda_{2}}{\lambda_{1}}$ as occurs for $t_{\text {lag }}$.

Figure 9 shows the effect of binding on $t_{\text {eff }}$ when $D_{v}=0$. The effective time constant decreases with an increase in the unbinding rate $\lambda_{1}$. For larger values of $D$, the influence of the unbinding rate $\lambda_{1}$ is less significant because diffusion becomes the driving mechanism. The time constant increases with the binding rate (Fig. 9). As $D$ increases, chemical effects play a minor role. The quantitative dependence of $t_{\text {eff }}$ on the unbinding rate is presented in Table 1. 

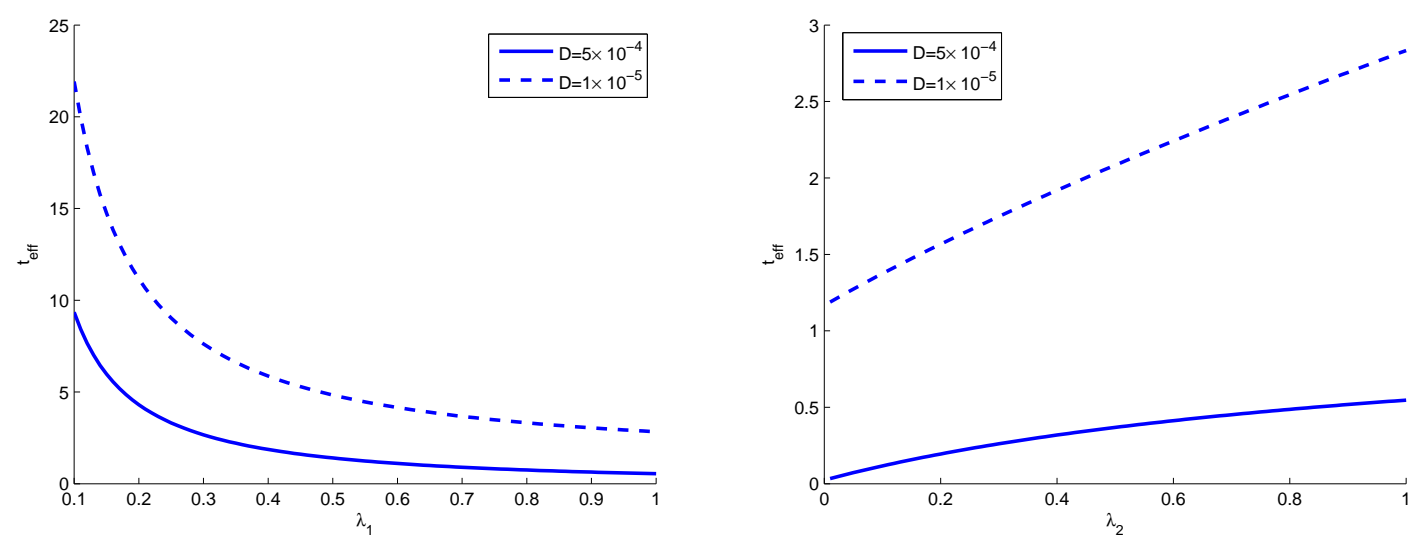

Figure 9: Influence of chemical reactions on $t_{\text {eff }}$ for two diffusion coefficients, with $\lambda_{2}=1$ (left) and $\lambda_{1}=1$ (right).

\begin{tabular}{|c|c|c|c|c|}
\hline & $\lambda_{1}=\lambda_{2}=10^{-3}$ & $\lambda_{1}=\lambda_{2}=0.1$ & $\lambda_{1}=10^{-3}, \lambda_{2}=0.1$ & $\lambda_{1}=0.1, \lambda_{2}=10^{-9}$ \\
\hline$t_{e f f}$ & 499.9851 & 5 & 990.1043 & $5.843 \times 10^{-5}$ \\
\hline
\end{tabular}

Table 2: Effective time constant for problem (26) for different values of $\lambda_{1}$ and $\lambda_{2}(\Omega=$ $\left.\left[0,10^{-3}\right], u_{0}=1, L=10^{-3}, D=3 \times 10^{-3}, D_{v}=10^{-6}, E_{0}=10^{3}, E_{1}=10\right)$.

In Figure 10, $t_{\text {eff }}$ is plotted as a function of $\lambda_{1}$ and $\lambda_{2}$. When $\lambda_{2}$ is held constant, $t_{\text {eff }}$ is a decreasing function of $\lambda_{1}$. The larger the unbound rate, the quicker the steady-state flux is achieved. Note that when $\lambda_{2}=0$, Eq. (25) is obtained.

Table 2 shows the effects of the reaction rates on $t_{\text {eff }}$. The effective time constant for the mechanistic model described by Eq. (1) is $5.833 \times 10^{-5}$. When $\frac{\lambda_{2}}{\lambda_{1}}=1, t_{e f f}$ is only a function of $\lambda_{1}$, as shown by Eq. (36). As $\lambda_{1}$ increases, the equilibrium state is reached more quickly. When $\frac{\lambda_{2}}{\lambda_{1}}<1$, the binding rate is smaller than the unbinding rate which promotes molecular transport. Chemical affinity always induces a delay when compared with diffusion through a membrane where only mechanical effects are modelled. For a small binding rate $\lambda_{2}, t_{\text {eff }}$ estimated from Eq. (36) approaches the value calculated from Eq. (25).

Equations (32) and (36) can be used to design systems with predefined steady-state fluxes. This approach leads to the estimation of the binding/unbinding rates, the diffusion coefficient or the Young modulus.

Some numerical results are shown in Tables 3 and 4 . The effective time constant and steady-state flux were a priori fixed (Table 3). The parameter $\lambda$ is such that $\lambda=\lambda_{1}=\lambda_{2}$. As the steady-state flux (32) in the second column is half value of the flux in the first column, we have 


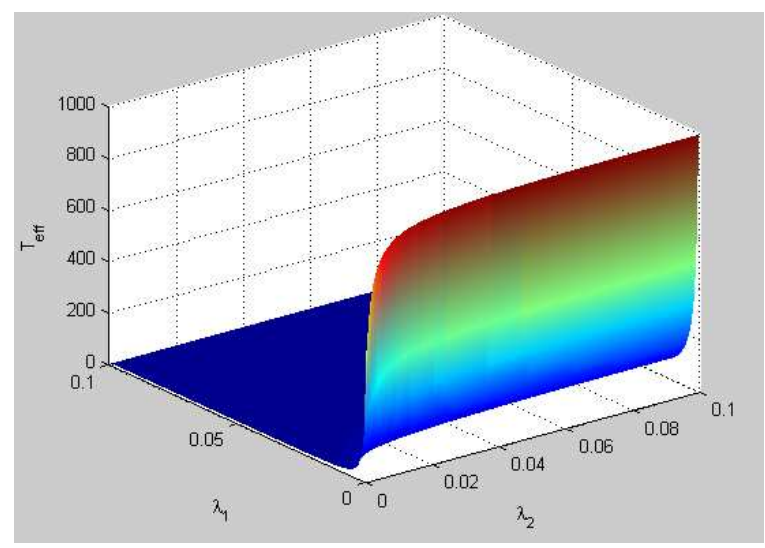

Figure 10: Influence of chemical reactions on $t_{\text {eff }}\left(\Omega=\left[0,10^{-3}\right], u_{0}=1, L=10^{-3}, D=\right.$ $\left.3 \times 10^{-3}, D_{v}=10^{-6}, E_{0}=10^{3}, E_{1}=10\right)$.

\begin{tabular}{|c|c|c|c|}
\hline & $t_{\text {eff }}=2, J^{s}=1$ & $t_{\text {eff }}=1, J^{s}=0.5$ & $t_{\text {eff }}=10, J^{s}=0.5$ \\
\hline \hline$\lambda$ & 0.25 & 0.5 & 0.05 \\
$D$ & $2 \times 10^{-3}$ & $1.5 \times 10^{-3}$ & $1.5 \times 10^{-3}$ \\
\hline
\end{tabular}

Table 3: Characterization of a membrane with a predefined flux profile $\left(u_{0}=1, L=\right.$ $\left.10^{-3}, \mu=10^{-5}, D_{v}=10^{-6}, E_{0}=10^{3}, E_{1}=10\right)$.

$$
D^{(2)}-D_{v} E_{0}=\frac{1}{2}\left(D^{(1)}-D_{v} E_{0}\right),
$$

where $D^{(i)}$ is the value of diffusion in column $(i), i=1,2$. From Eq. (37) we obtain

$$
D^{(2)}=\frac{1}{2} D^{(1)}+\frac{1}{2} D_{v} E_{0}
$$

The diffusion is larger than $\frac{1}{2} D^{(1)}$ because mechanical effects are present. If $D_{v}=0$, we have $D^{(1)}=\frac{1}{2} D^{(2)}$. The common value $\lambda$ of the reaction rates determines the effective time constant.

As $\frac{\lambda_{2}}{\lambda_{1}}=1$, an unbinding rate larger than the one listed in column 1 must be considered to obtain a smaller effective time constant (in column 2). The diffusion coefficients in the

\begin{tabular}{|c|c|c|c|}
\hline & $t_{\text {eff }}=2, J^{s}=2 \times 10^{-4}$ & $t_{\text {eff }}=2, J^{s}=2$ & $t_{\text {eff }}=10, J^{s}=0.5$ \\
\hline \hline$\lambda$ & 0.6 & 0.25 & 0.05 \\
$E_{0}$ & 3000 & 1000 & 2500 \\
\hline
\end{tabular}

Table 4: Mechanical and chemical characterization of a chemical and mechanical properties of a membrane with a predefined flux profile $\left(u_{0}=1, L=10^{-3}, \mu=10^{-5}, D=3 \times 10^{-3}, D_{v}=\right.$ $\left.10^{-6}, E_{1}=10\right)$. 
second and third columns are equal because the flux is the same in both cases. As $\lambda$ decreases to 0.05 , it takes a longer time to reach the steady-state flux (Column 3).

In Table 4, values of $\lambda$ and $E_{0}$ are selected such that the predefined values for the effective time constant and steady-state flux are achieved. The Young modulus $E_{0}$ of the free spring is chosen so that the steady-state flux satisfies Eq. (32). As $E_{0}$ decreases, $t_{\text {eff }}$ and the stress exerted by the polymer on the penetrant decrease. Considering that in column 2 effective time is the same as in column 1 , the value of the unbinding rate must decrease. A similar trend is observed in column 3 .

\section{Conclusions}

The coupled effect of mechanical and chemical properties on the permeation of a fluid in a polymeric matrix was considered. A generalized Maxwell model was applied to describe relaxation times of large and small molecules. Closed formulas, which included the material physical properties, were established for the lag time and effective time constant. These expressions would allow researchers to specify properties that lead to a target steady-state flux in a predefined time interval. The Young modulus of the isolated spring has a significant influence on the effective time constant and the steady-state flux while the viscosity has only a negligible impact. The Young modulus of a polymer can be changed by blending together, different polymers, or connecting individual chains by cross-links. In this case, the chains lose their initial mobility and become less elastic. The Young modulus increases and the steady flux decreases. The lag time and effective time constant increase. The inclusion of binding sites or the use of polymers with affinity for the diffusing fluid induce a delay in the permeation. While these findings are expected, this work makes it possible to tune the mechanical and chemical properties to achieve desired transport characteristics. 


\section{A Annex}

\begin{tabular}{|l|l|}
\hline Symbol & Definition (unities) \\
\hline$u$ & concentration of the penetrant $\left(\mathrm{mol} / \mathrm{m}^{3}\right)$ \\
$D$ & diffusion coefficient $\left(\mathrm{m}^{2} / \mathrm{s}\right)$ \\
$D_{v}$ & stress-driven diffusion coefficient $(\mathrm{mol} /(\mathrm{m} . \mathrm{s} . \mathrm{Pa}))$ \\
$\sigma$ & stress $(\mathrm{Pa})$ \\
$E_{0}$ & Young modulus $(\mathrm{Pa})$ \\
$E_{i}$ & Young modulus of arms $(\mathrm{Pa})$ \\
$\epsilon$ & strain \\
$\tau_{i}$ & relaxation time $(\mathrm{s})$ \\
$\mu_{i}$ & viscosity $(\mathrm{Pa} . \mathrm{s})$ \\
$J$ & flux $\left(\mathrm{mol} /\left(\mathrm{m}^{2} . s\right)\right)$ \\
$\lambda_{1}$ & unbinding rate $\left(\mathrm{s}^{-1}\right)$ \\
$\lambda_{2}$ & binding rate $\left(\mathrm{s}^{-1}\right)$ \\
$L$ & length of the polymeric matrix $(\mathrm{m})$ \\
$J^{s}$ & steady-state flux $\left(\mathrm{mol} /\left(\mathrm{m}^{2} . s\right)\right)$ \\
$t_{\text {eff }}$ & effective time $(s)$ \\
$t_{l a g}$ & lag time $(s)$ \\
\hline
\end{tabular}

\section{References}

[1] R. Gaspar, R. Duncan, Polymeric carriers: preclinical safety and the regulatory implications for design and development of polymer therapeutics, Adv Drug Deliv Rev., 12, 61, 1220-31, 2009.

[2] M. Jelvehgari, H. Valizadeh, F. Kiafarab, L. Afandipourc, Taste Masking and Characterization of Chlorpheniramine. Maleate by Using Enteric Polymers Carrier System, Journal of Reports in Pharmaceutical Sciences, 2(1), 45-58, 2013.

[3] G. Thilagavathi, A.S.M. Raja, T. Kannaian, Nanotechnology and Protective Clothing for Defence Personnel, Defence Science Journal, Vol. 58, No. 4, 451-459, 2008.

[4] C. F. C. M. Fong, C. Moresoli, S. Xiao, Y. Li, J. Bovenkamp, D. De Kee, Modeling diffusion through geomembranes, J. Appl. Polym. Sci., 67, 1885-1889, 1998.

[5] J.A. Ferreira, P. de Oliveira, P.M. da Silva, L. Simon, Flux tracking in delivery polymeric systems, Appl. Math. Modelling, 35, 4684-4696, 2011.

[6] R.A. Siegel, Characterization of relaxation to steady state in membranes with binding and reaction, J. Memb. Sci., 251, 91-99, 2005.

[7] R.A. Siegel, E.L. Cussler, Reactive barrier membranes: some theoretical observations regarding the time lag and breakthrough curves, J. Memb. Sci., 229, 33-41, 2004.

[8] H.F. Brinson, L.C. Brinson, Polymer Engineering Science and Viscoelasticity, An Introduction, Springer, 2008.

[9] L. Simon, Analysis of heat-aided membrane controlled drug released from a process control prespective, Int. J. Heat Mass Transfer, 50, 2425-2433, 2007. 
[10] A. Strzelewicz, Z. J. Grzywna, On the permeation time lag for different transport equations by Frisch method, J. Memb. Sci., 322, 460-465, 2008.

[11] J. Crank. The mathematics of diffusion Oxford University Press, 2 edition, 1980.

[12] H.L. Frisch. The Time Lag in Diffusion, J. Phys. Chem., 61 , 93-95, 1957.

[13] J.A. Ferreira, M. Grassi, E. Gudino, P. de Oliveira, A new look to non-Fickian diusion, preprint Universidade de Coimbra, 2013, submitted.

[14] J.A. Ferreira, M. Grassi, E. Gudino, P. de Oliveira, A mathematical model for controlled drug delivery in swelling polymers, Proceedings of International Conference on Computational and Mathematical Methods in Science and Engineering, Almería, Spain, June 24-27, I. Halmiton, J. Vigo-Aguiar (Edt), 630-641, 2013.

[15] Q. Liu, X. Wang, D. De Kee, Mass transport through swelling membranes, Int. J. Eng. Sci., 43, 1464-1470, 2005.

[16] S. Xiao, C. Moresoll, J. Bovenkamp, D. De Kee, Sorption and permeation of organic environmental contaminants through PVC geomembranes, J. Appl. Polym. Sci., 63, 1189-1197, 1997.

[17] D.A. Edwards, D.S. Cohen, A mathematical model for a dissolving polymer, AIChE J. 18, 2345$2355,1995$.

[18] D.A. Edwards, R. Cairncross, Desorption overshoot in polymer-penetrant systems: asymptotic and computational results, SIAM J. Appl. Math., 63(1), 98-115, 2002.

[19] L. Simon, Timely drug delivery from controlled-release devices: Dynamic analysis and novel design concepts, Math. Biosci., 217, 151-158, 2009.

[20] R. Collins, The choice of an effective time constant for diffusive processes in finite systems, J. Phys. D Appl. Phys. 13, 19-37, 1980.

[21] C.L. Huang, T.W.J. Steele, E. Widjaja, F.Y.C. Boey, S.S. Venkatraman, J.S.C. Loo, The influence of additives in modulating drug delivery and degradation of PLGA thin film, NPG Asia Materials, $5,1-11,2013$.

[22] L. Simon, Control of Biological and Drug-Delivery Systems, John Wiley and Sons,New Jersey, USA, 2013.

[23] J.A. Ferreira, P. de Oliveira, P. da Silva, Analytics and numerics of drug release tracking, J. of Comput. and Appl. Math. 236, 3572-3583, 2012. 